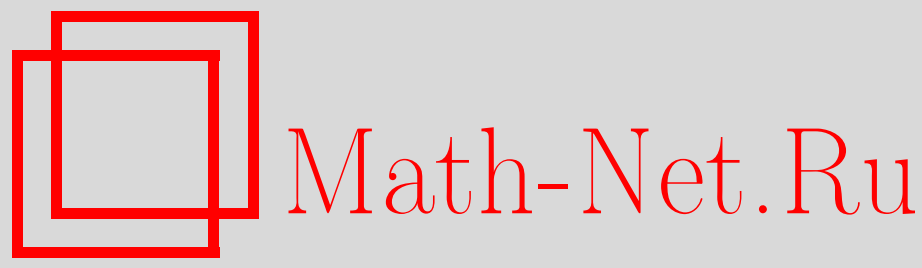

Д. Коледа, О способе равномерного упорядочения вещественных алгебраических чисел, Совр. пробл. матем., 2016, выпуск 23, 69-78

DOI: https://doi.org/10.4213/spm62

Использование Общероссийского математического портала Math-Net.Ru подразумевает, что вы прочитали и согласны с пользовательским соглашением http://www . mathnet.ru/rus/agreement

Параметры загрузки:

IP: 54.162 .127 .20

26 апреля 2023 г., 11:19:50 


\title{
О способе равномерного упорядочения вещественных алгебраических чисел
}

\author{
Д. Коледа \\ Институт математики НАН Беларуси \\ koledad@rambler.ru
}

\section{1. Введение}

Статья посвящена распределению алгебраических чисел на вещественной прямой и основана на докладе автора на конференции памяти Анатолия Алексеевича Карацубы в янваpe 2016 г.

Чтобы избежать разночтений начнем с основных определений и соглашений, которые используются при изложении.

Под иелочисленным многочленом будем понимать многочлен с целыми коэффициентами. Алгебраическим числом называется число $\alpha$, для которого существует ненулевой целочисленный многочлен $p$ такой, что $p(\alpha)=0$. Минимальным многочленом алгебрачческого числа $\alpha$ будем называть целочисленный многочлен $p$ наименьшей степени со взаимно простыми коэффициентами и положительным старшим коэффициентом такой, что $p(\alpha)=0$. Различные алгебраические числа $\alpha_{1}, \alpha_{2}, \ldots, \alpha_{k}$ называются (алгебраически) сопряженными, если они имеют один и тот же минимальный многочлен.

Пусть

$$
p(x)=a_{n} x^{n}+\cdots+a_{1} x+a_{0} .
$$

Высотой многочлена $H(p)$ будем называть величину

$$
H(p):=\max _{0 \leqslant i \leqslant n}\left|a_{i}\right|
$$

Степень алгебраического числа $\operatorname{deg} \alpha$ определяется как степень его минимального многочлена, а высота $H(\alpha)$ - как высота его минимального многочлена.

В статье будем полагать, что степень алгебраических чисел и многочленов фиксирована и равна $n$; высоты алгебраических чисел и многочленов ограничены сверху параметром $Q$; асимптотические формулы рассматриваются при $Q \rightarrow+\infty$; неявные постоянные не зависят от $Q$, но могут зависеть от $n$.

Необходимо отметить, что кроме цитируемых ниже в обзоре работ есть большое количество публикаций, посвященных подсчету числа элементов в специальных классах алгебраических чисел, например, алгебраических чисел на комплексной плоскости, целых алгебраических чисел и т.д. Зачастую в подобных результатах используются другие высоты, в частности, так называемая высота Вейля. В нашем обзоре мы ограничимся исключительно распределением вещественных алгебраических чисел относительно равномерной высоты.

1.1. Рациональные числа. Наипростейшими алгебраическими числами являются числа рациональные. С ними связано множество классических задач, большинство которых формулируется в терминах последовательностей (или так называемых рядов) Фарея. Последовательностъю Фарея порядка $Q$ называется упорядоченная по возрастанию последовательность

Работа выполнена при финансовой поддержке CRC 701 (университет г. Билефельда, Германия). 
несократимых рациональных дробей со знаменателем не более $Q$, лежащих на $[0,1]$ :

$$
\mathcal{F}_{Q}:=\left\{\frac{a}{b}: a, b \in \mathbb{Z}, 0 \leqslant a \leqslant b \leqslant Q, \operatorname{gcd}(a, b)=1\right\} .
$$

В 1963 г. Вальфиш [1] доказал, что

$$
\# \mathcal{F}_{Q}=\frac{3}{\pi^{2}} Q^{2}+O\left(Q(\log Q)^{2 / 3}(\log \log Q)^{4 / 3}\right) .
$$

Известно, что классическая последовательность Фарея распределена равномерно на $[0,1]$, т.е. для любого промежутка $I \subseteq[0,1]$ верно равенство

$$
\lim _{Q \rightarrow \infty} \frac{\#\left(\mathcal{F}_{Q} \cap I\right)}{\# \mathcal{F}_{Q}}=|I| .
$$

Доказательство этого факта можно найти, например, в [2]-[4]. Кроме того, распределение последовательностей Фарея тесно связано с гипотезой Римана [5].

«Неидеальность» распределения Фареевских последовательностей (по сравнению с непрерывным равномерным на $[0,1]$ распределением) характеризуется отклонением (discrepancy), определяемым как

$$
D_{Q}:=\sup _{\alpha \in[0,1]}\left|\frac{\#\left(\mathcal{F}_{Q} \cap[0, \alpha]\right)}{\# \mathcal{F}_{Q}}-\alpha\right| .
$$

В 1973 г. Нидэррайтэр [6] установил, что $D_{Q} \asymp Q^{-1}$. В 1999 г. Дресс [7] доказал точное равенство $D_{Q}=Q^{-1}$.

Много интересных фактов о последовательностях Фарея можно найти в обзоре [8].

1.2. Алгебраические числа на вещественной прямой. В 1970 г. Бэйкер и Шмидт [9] ввели понятие регулярной системы и установили, что алгебраические числа образуют регулярную систему (параметры этой системы были не оптимальны).

За результатами [9] последовал ряд их усилений в работах Берника [10], [11], Берника и Додсона [12], а в 1999 г. Бересневич [13] доказал регулярность алгебраических чисел с неулучшаемыми параметрами.

Относительно простыми словами без потери строгости результат Бересневича можно сформулировать следующим образом.

Теорема 1 [13]. Существует постоянная $c_{n}$, зависящая только от $n$ такая, что на любом промежутке $I \subseteq[-1,1]$ для всех достаточно больших $Q \in \mathbb{N}$ существует не менее

$$
c_{n}|I| Q^{n+1}
$$

алгебрачческих чисел $\alpha_{1}, \ldots, \alpha_{k}$ степени не более $n$ и высоты не более $Q$, таких что

$$
\left|\alpha_{i}-\alpha_{j}\right| \geqslant Q^{-n-1}, \quad 1 \leqslant i<j \leqslant k .
$$

Общий обзор результатов о регулярных системах алгебраических чисел и их приложениях к проблемам вычисления размерности Хаусдорфа можно найти в [12] и [14].

В 1971 г. Браун и Малер [15] предложили обобщение классических последовательностей Фарея на алгебраические числа произвольной степени: последовательностъю Фарея степени $n$ порядка $Q$ называется последовательность всех вещественных корней всех целочисленных многочленов степени $n$ и высоты не более $Q$.

В 1985 г. К. Малер в письме В.Г. Спринджуку писал, что даже для второй степени не известно, как распределены алгебраические числа. 
Пусть $\mathbb{A}_{n}$ - множество алгебраических чисел степени $n$. Зафиксируем $n \geqslant 2$ и рассмотрим множество $I \subset \mathbb{R}$. Обозначим через $\Phi(Q, I)$ количество алгебраических чисел $\alpha \in I$ с $\operatorname{deg}(\alpha)=n$ и $H(\alpha) \leqslant Q$ :

$$
\Phi(Q, I)=\#\left\{\alpha \in \mathbb{A}_{n} \cap I: H(\alpha) \leqslant Q\right\}
$$

При $n=2$ далее в формулах появляется дополнительный множитель $\log Q$, поэтому для краткости используется символ $l(n)$, определенный как

$$
l(n):= \begin{cases}1, & n \leqslant 2 \\ 0, & n \geqslant 3 .\end{cases}
$$

В серии работ [16]-[18] была доказана следующая теорема.

Теорема 2. Пусть $n \in \mathbb{N}$ фиксировано. Пусть $I \subseteq \mathbb{R}-$ промежуток. Тогда при $Q \rightarrow \infty$ верна формула

$$
\Phi(Q, I)=\frac{Q^{n+1}}{2 \zeta(n+1)} \int_{I} \phi_{n}(x) d x+O\left(Q^{n} \log ^{l(n)} Q\right),
$$

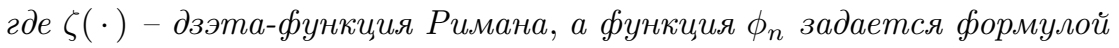

$$
\phi_{n}(x)=\int_{B_{n}(x)}\left|\sum_{k=1}^{n} k t_{k} x^{k-1}\right| d t_{1} \ldots d t_{n}
$$

с интегрированием по области

$$
B_{n}(x)=\left\{\left(t_{1}, \ldots, t_{n}\right) \in \mathbb{R}^{n}: \max _{1 \leqslant k \leqslant n}\left|t_{k}\right| \leqslant 1,\left|\sum_{k=1}^{n} t_{k} x^{k}\right| \leqslant 1\right\} .
$$

$B(3)$ неявная постоянная в символе $O(\cdot)$ зависит только от $n$.

При этом для бесконечного числа промежутков $I=I(Q)$ порядок остаточного члена в (3) не менее чем $Q^{n}$.

Кроме того, функция $\phi_{n}(x)$ удовлетворяет следующим функциональным уравнениям:

$$
\phi_{n}(-x)=\phi_{n}(x), \quad \phi_{n}\left(\frac{1}{x}\right)=x^{2} \phi_{n}(x) .
$$

При $|x| \leqslant 1-1 / \sqrt{2}$ интеграл (4) равен (см. [18])

$$
\phi_{n}(x)=\frac{2^{n-1}}{3}\left(3+\sum_{k=1}^{n-1}(k+1)^{2} x^{2 k}\right)
$$

Функция $\phi_{n}$ с точностью до постоянного множителя совпадает с плотностью распределения вещественных нулей случайного многочлена с независимыми равномерно распределенными на $[-1,1]$ коэффициентами [19].

Для $n=1$, т.е. для рациональных чисел, как уже отмечалось выше, асимптотическая формула (3) была по существу известна, с тем лишь отличием, что в классических результатах рассматривались $I \subseteq[0,1]$. Функция $\phi_{1}(x)$ имеет вид

$$
\phi_{1}(x)=\frac{1}{\max \left(1, x^{2}\right)} .
$$

Как следствие из результатов Нидэррайтэра [6] и Дресса [7] можно доказать следующее утверждение [18]. 
Теорема 3 (см. раздел 4 в [18]). Пусть $n=1$. Тогда

$$
\sup _{I \subseteq \mathbb{R}}\left|\frac{\Phi(Q, I)}{\Phi(Q, \mathbb{R})}-\frac{1}{4} \int_{I} \phi_{1}(x) d x\right| \asymp \frac{1}{Q},
$$

где точная верхняя грань берется по всем промежуткам вещественной оси, а неявная по-

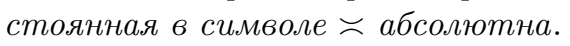

Отсюда, учитывая оценку (1) количества дробей Фарея и равенство $\Phi(Q, \mathbb{R})=4 \# \mathcal{F}_{Q}-5$ (при $n=1)$, можно получить более точную оценку остаточного члена в $(3)$.

Теорема 4 [18]. При $n=1$ остаточный член в (3) имеет вид

$$
O\left(Q(\log Q)^{2 / 3}(\log \log Q)^{4 / 3} \int_{I} \frac{d x}{\max \left(1, x^{2}\right)}+Q\right)
$$

где неявная постоянная абсолютна.

Отметим, что интеграл в $(7)$ стремится к нулю при $|I| \rightarrow 0$, соответственно для коротких промежутков логарифм фактически исчезает.

Похожее уточнение доказано [20] и для алгебраических чисел второй степени.

Tеорема 5 [20]. При $n=2$ остаточный член в (3) имеет вид

$$
O\left(Q^{2} \log Q \int_{I} \frac{d x}{\max \left(1, x^{2}\right)}+Q^{2}\right)
$$

где неявная постоянная абсолютна.

1.3. Сопряженные алгебраические числа. Уже для второй степени возникает задача о совместном распределении вещественных алгебраически сопряженных чисел.

В 2010 г. Бересневич, Берник и Гётце [21] установили оценку снизу для числа пар близких сопряженных вещественных алгебраических чисел $n$-й степени на заданном промежутке.

Теорема 6 [21]. Для $n \geqslant 2 u 0<\gamma \leqslant(n+1) / 3$ при достаточно больиих $Q$ в любом промежутке $I \subset[-1 / 2,1 / 2]$ существует не менее

$$
\frac{1}{2} Q^{n+1-2 \gamma}|I|
$$

вещественных алгебраических чисел $\alpha$ степени $\operatorname{deg}(\alpha)=n$ с высотой $H(\alpha) \asymp_{n} Q$, у каждого из которых есть хотя бы одно вещественное сопряженное алгебрачческое число $\alpha^{*}$ такое, чmo

$$
\left|\alpha-\alpha^{*}\right| \asymp_{n} Q^{-\gamma}
$$

Для формулировки следующей теоремы потребуются некоторые дополнительные обозначения.

Пусть $\mathbf{x}:=\left(x_{1}, \ldots, x_{k}\right) \in \mathbb{R}^{k}$. Элементарные симметричные многочлены от $\mathbf{x}$ степени $m$ будем обозначать так:

$$
\sigma_{m}(\mathbf{x}):= \begin{cases}1, & \text { если } m=0, \\ \sum_{1 \leqslant j_{1}<\cdots<j_{m} \leqslant k} x_{j_{1}} x_{j_{2}} \ldots x_{j_{m}}, & \text { если } 1 \leqslant m \leqslant k, \\ 0 & \text { в остальных случаях. }\end{cases}
$$


Пусть $\mathbb{A}_{n}^{(k)}$ есть множество $k$-векторов $\left(\alpha_{1}, \alpha_{2}, \ldots, \alpha_{k}\right) \in \mathbb{R}^{k}$, где $\alpha_{1}, \alpha_{2}, \ldots, \alpha_{k}$ - вещественные сопряженные алгебраические числа степени $n$, т.е. различные корни одного и того же неприводимого над $\mathbb{Q}$ целочисленного многочлена степени $n$.

Пусть $B \subset \mathbb{R}^{k}$. Обозначим через $\Phi_{k}(Q, B)$ количество векторов из $\mathbb{A}_{n}^{(k)} \cap B$ с высотой координат $\leqslant Q$ :

$$
\Phi_{k}(Q, B)=\#\left\{\left(\alpha_{1}, \alpha_{2}, \ldots, \alpha_{k}\right) \in \mathbb{A}_{n}^{(k)} \cap B: H\left(\alpha_{i}\right) \leqslant Q\right\} .
$$

В 2015 г. Гётце, Запорожец и Коледа доказали следующую теорему.

ТеОРема 7 [22]. Пусть В - ограниченная область в $\mathbb{R}^{k}$ с границей, состоящей из конечного числа m алгебрачческих поверхностей, максимальная степень которых не превосходит $d$. Тогда

$$
\Phi_{k}(Q, B)=\frac{(2 Q)^{n+1}}{2 \zeta(n+1)} \int_{B} \rho_{k}(\mathbf{x}) d \mathbf{x}+O\left(Q^{n} \log ^{l(n)} Q\right), \quad Q \rightarrow \infty,
$$

где функиия $\rho_{k}$ определяется формулой

$$
\rho_{k}(\mathbf{x})=2^{-n-1} \prod_{1 \leqslant i<j \leqslant k}\left|x_{i}-x_{j}\right| \int_{D_{\mathbf{x}}} \prod_{i=1}^{k}\left|\sum_{j=0}^{n-k} t_{j} x_{i}^{j}\right| d t_{0} \ldots d t_{n-k}
$$

с интегрированием по области $D_{\mathbf{x}}$, заданной как

$$
D_{\mathbf{x}}=\left\{\left(t_{0}, \ldots, t_{n-k}\right) \in \mathbb{R}^{n-k+1}: \max _{0 \leqslant i \leqslant n}\left|\sum_{j=0}^{n-k}(-1)^{i-j} \sigma_{i-j}(\mathbf{x}) t_{j}\right| \leqslant 1\right\} .
$$

Неявная постоянная в остаточном члене в (8) зависит только от $n, m u d$.

Функция (9) совпадает с корреляционной функцией корней случайного многочлена с независимыми равномерно распределенными на $[-1,1]$ коэффициентами (см. [19] и [23]).

В двух частных случаях формула (9) существенно упрощается:

а) при $k=1$ формула (9) фактически превращается в (4) и описывает распределение алгебраических чисел на вещественной прямой, т.е.

$$
\rho_{1}(x)=2^{-n-1} \phi_{n}(x)
$$

б) при $k=n$ теорема 7 описывает распределение вполне вещественных алгебраических чисел; при этом функция $\rho_{n}$ принимает вид

$$
\rho_{n}(\mathbf{x})=\frac{2^{-n}}{(n+1)}\left(\frac{1}{\max _{0 \leqslant i \leqslant n}\left|\sigma_{i}(\mathbf{x})\right|}\right)^{n+1} \prod_{1 \leqslant i<j \leqslant n}\left|x_{i}-x_{j}\right| .
$$

Функция $\rho_{k}$ удовлетворяет следующим функциональным уравнениям:

а) для любой перестановки $s$ длины $k$

$$
\rho_{k}\left(x_{s(1)}, x_{s(2)}, \ldots, x_{s(k)}\right)=\rho_{k}(\mathbf{x}) ;
$$

б) для любых $\mathbf{x} \in \mathbb{R}^{k}$

$$
\rho_{k}(-\mathbf{x})=\rho_{k}(\mathbf{x})
$$

в) для любых $\mathbf{x} \in \mathbb{R}^{k}$ с ненулевыми координатами

$$
\rho_{k}\left(x_{1}^{-1}, x_{2}^{-1}, \ldots, x_{k}^{-1}\right)=\rho_{k}(\mathbf{x}) \prod_{i=1}^{k} x_{i}^{2} .
$$




\section{2. О равномерном упорядочении}

На конференции после доклада автора Николай Германович Мощевитин задал вопрос: известно ли автору какое-то конкретное упорядочение алгебраических чисел, чтобы полученная последовательность была распределена равномерно? Из общих соображений можно доказать, что любую всюду плотную последовательность можно переупорядочить так, чтобы получилось равномерное распределение [4; § 2.4]. Однако в данном случае естественно потребовать от такого упорядочения, чтобы оно учитывало внутренние свойства алгебраических чисел, в частности единообразно действовало на сопряженные алгебраические числа. Предложенное ниже упорядочение не согласовано с алгебраическим сопряжением, хотя существенно опирается на статистические свойства вещественных алгебраических чисел относительно равномерной высоты.

Пусть $\psi$ - положительная функция, удовлетворяющая условию Липшица

$$
|\psi(x)-\psi(y)| \leqslant C|x-y| \quad \text { для всех } \quad x, y \in I_{0}=[-R, R] \text {, }
$$

где $R \geqslant 1$ - фиксированное число. Введем обозначение

$$
\Phi^{*}(Q, I)=\#\left\{\alpha \in \mathbb{A}_{n} \cap I: \psi(\alpha) H(\alpha) \leqslant Q\right\}
$$

Ниже мы покажем, как с помощью выбора функции $\psi$ можно добиться равномерности распределения чисел, учтенных в $\Phi^{*}(Q, I)$. А именно, докажем следующую теорему.

Теорема 8. Пусть $\psi(x)=\phi_{n}(x)^{1 /(n+1)}$, где $\phi_{n}$ определена в (4). Тогда

$$
\Phi^{*}(Q, I)=\frac{Q^{n+1}}{2 \zeta(n+1)}|I|+O\left(R^{n /(n+1)} Q^{n+1 / 2} \log ^{l(n) / 2} Q \cdot|I|+R^{2 n /(n+1)} Q^{n} \log ^{l(n)} Q\right),
$$

где неявная постоянная в символе $O(\cdot)$ зависит только от $n$.

ЗАмечаниЕ. Функцию $\psi(\alpha) H(\alpha)$ можно было бы толковать как некоторую «высоту» алгебраического числа $\alpha$, однако при таком определении функции $\psi$, как в теореме 8 , сопряженные алгебраические числа, если у них разные абсолютные величины, будут иметь, вообще говоря, разные «высоты».

Для доказательства теоремы 8 нам потребуется пара вспомогательных утверждений. Необходимые факты о $\phi_{n}$ для удобства соберем в одну лемму.

ЛЕмма 1. Функиия $\phi_{n}$, определенная в (4), обладает следующими свойствами:

I) для всех $x \in \mathbb{R}$ выполняется неравенство

$$
\frac{C_{1}}{\max \left(1, x^{2}\right)} \leqslant \phi_{n}(x) \leqslant \frac{C_{2}}{\max \left(1, x^{2}\right)},
$$

где $C_{1}$ и $C_{2}$ - положительные постоянные, зависящие только от $n$ :

$$
C_{1}=\min _{x \in[0,1]} \phi_{n}(x), \quad C_{2}=\max _{x \in[0,1]} \phi_{n}(x)
$$

II) для любых $x, y \in \mathbb{R}$ таких, что $0 \leqslant y-x \leqslant 1, u$ любого $\theta \in[x, y]$ верно

$$
\left|\phi_{n}(x)-\phi_{n}(y)\right| \leqslant \frac{C_{3}|x-y|}{\max \left(1,|\theta|^{3}\right)}
$$

где $C_{3}$ - положительная постоянная, которая зависит только от $n$. 
ДокАзАТЕЛьство. Несложно заметить, что функция $\phi_{n}$ непрерывна и ограничена на $[0,1]$, и, следовательно, достигает там своих максимального и минимального значений. Из уравнений (5) сразу же получаем свойство I).

Докажем II). Обозначим для краткости $F(\mathbf{t} ; x)=\left|\sum_{k=1}^{n} k t_{k} x^{k-1}\right|$. Тогда

$$
\phi_{n}(x)-\phi_{n}(y)=\int_{B_{n}(x)}(F(\mathbf{t} ; x)-F(\mathbf{t} ; y)) d \mathbf{t}+\int_{B_{n}(x)} F(\mathbf{t} ; y) d \mathbf{t}-\int_{B_{n}(y)} F(\mathbf{t} ; y) d \mathbf{t} .
$$

Пусть $x, y \in[-2,2]$. Нетрудно показать, что для любых $\mathbf{t} \in[-1,1]^{n}$ функция $F(\mathbf{t} ; x)$ ограничена постоянной величиной, зависящей только от $n$, и

$$
|F(\mathbf{t} ; x)-F(\mathbf{t} ; y)| \leqslant C|x-y|
$$

где $C$ - постоянная, зависящая только от $n$.

Также можно показать, что при $x, y \in[-2,2]$ мера симметрической разности множеств $B_{n}(x)$ и $B_{n}(y)$ удовлетворяет неравенству

$$
\operatorname{mes}_{n}\left(B_{n}(x) \ominus B_{n}(y)\right) \leqslant C^{\prime}|x-y|,
$$

где $C^{\prime}$ - постоянная, зависящая только от $n$.

Собирая все вместе, получаем при $x, y \in[-2,2]$

$$
\left|\phi_{n}(x)-\phi_{n}(y)\right| \leqslant \widetilde{C}|x-y|
$$

для некоторой зависящей только от $n$ постоянной $\widetilde{C}$.

Пусть теперь $x, y \in[1,+\infty)$. Тогда, пользуясь вторым уравнением в $(5)$, получаем

$$
\begin{aligned}
\left|\phi_{n}(x)-\phi_{n}(y)\right| & =\left|\frac{1}{x^{2}} \phi_{n}\left(\frac{1}{x}\right)-\frac{1}{y^{2}} \phi_{n}\left(\frac{1}{y}\right)\right| \\
& \leqslant \frac{1}{x^{2}}\left|\phi_{n}\left(\frac{1}{x}\right)-\phi_{n}\left(\frac{1}{y}\right)\right|+\phi_{n}\left(\frac{1}{y}\right)\left|\frac{1}{x^{2}}-\frac{1}{y^{2}}\right| \leqslant|x-y|\left(\frac{\widetilde{C}}{x^{3} y}+C_{2} \frac{(x+y)}{(x y)^{2}}\right) .
\end{aligned}
$$

Объединяя оба случая в одном неравенстве и подбирая постоянную $C_{3}$, получаем свойство II). Отметим, что такая свобода в выборе промежуточной точки $\theta$ возможна благодаря ограничению $|x-y| \leqslant 1$. Лемма доказана.

Лемма 2. Пусть $I \subset \mathbb{R}-$ конечный промежуток. Существует такое $\xi \in I$, что справедливо равенство

$$
\Phi^{*}(Q, I)=\Phi\left(\frac{Q}{\psi(\xi)}, I\right)+O\left(\frac{Q^{n}}{\psi(\xi)^{n}} \log ^{l(n)} Q\right)
$$

где функиия $\Phi(Q, I)$ определена в $(2)$; неявная постоянная в символе $O(\cdot)$ зависит только om $n$.

ДокАзАтЕльство. Скобками $\lfloor\cdot\rfloor$ будем обозначать округление до целого с недостатком. По определению $H(\alpha) \in \mathbb{N}$.

Пусть $m=\inf _{x \in I} \psi(x), M=\sup _{x \in I} \psi(x)$. Тогда

$$
\Phi\left(\frac{Q}{M}, I\right) \leqslant \Phi^{*}(Q, I) \leqslant \Phi\left(\frac{Q}{m}, I\right) .
$$

а) Если $Q / M \geqslant\lfloor Q / m\rfloor$, то в качестве $\xi$ можно взять любую точку из $I$, поскольку для любого $X \in[m, M]$ будет справедливо равенство

$$
\Phi^{*}(Q, I)=\Phi\left(\frac{Q}{X}, I\right)=\Phi\left(\left\lfloor\frac{Q}{X}\right\rfloor, I\right)
$$


б) Пусть $\lfloor Q / M\rfloor<\lfloor Q / m\rfloor$. Определим

$$
h=\max \left\{r \in \mathbb{Z}: \Phi(r, I) \leqslant \Phi^{*}(Q, I),\left\lfloor\frac{Q}{M}\right\rfloor \leqslant r<\left\lfloor\frac{Q}{m}\right\rfloor\right\} .
$$

В силу монотонности $\Phi(Q, I)$ по параметру $Q$ имеем

$$
\Phi(h, I) \leqslant \Phi^{*}(Q, I) \leqslant \Phi(h+1, I) .
$$

При этом для любого $\widetilde{h} \in[h, h+1)$ будет верно $\Phi(\widetilde{h}, I)=\Phi(h, I)$.

По теореме 2

$$
\Phi(h+1, I)-\Phi(h, I)=O\left(h^{n} \log ^{l(n)} h\right) .
$$

Таким образом, в качестве $\xi$ можно взять любую точку из $I$ такую, что $Q / \psi(\xi) \in[h, h+1]$.

Отметим, что в случае других определений высоты алгебраического числа высота, вообе говоря, не целое число. Однако для доказательства леммы 2 существенным обстоятельством является только дискретность множества значений высоты, что автоматически выполняется для любой функции высоты $\mathcal{H}(\cdot)$ со свойством

$$
\#\left\{\alpha \in \mathbb{A}_{n}: \mathcal{H}(\alpha) \leqslant X\right\}<\infty \quad \text { для всех } \quad X>0 .
$$

Перейдем к доказательству основной теоремы пункта. Разобьем промежуток $I$ на конечное число непересекающихся подпромежутков $I_{i}$. Тогда

$$
\Phi^{*}(Q, I)=\sum_{i} \Phi^{*}\left(Q, I_{i}\right)
$$

По теореме 2 и лемме 2 существует такое $\xi_{i} \in I_{i}$, что

$$
\Phi^{*}\left(Q, I_{i}\right)=\frac{1}{2 \zeta(n+1)}\left(\frac{Q}{\psi\left(\xi_{i}\right)}\right)^{n+1} \int_{I_{i}} \phi_{n}(x) d x+O\left(\left(\frac{Q}{\psi\left(\xi_{i}\right)}\right)^{n} \log ^{l(n)} Q\right),
$$

где неявная постоянная в символе $O(\cdot)$ зависит только от $n$.

По теореме Лагранжа существует $\eta_{i} \in I_{i}$ такое, что

$$
\int_{I_{i}} \phi_{n}(x) d x=\phi_{n}\left(\eta_{i}\right)\left|I_{i}\right|
$$

Таким образом,

$$
\Phi^{*}(Q, I)=\frac{Q^{n+1}}{2 \zeta(n+1)} \sum_{i} \frac{\phi_{n}\left(\eta_{i}\right)}{\psi\left(\xi_{i}\right)^{n+1}}\left|I_{i}\right|+O\left(Q^{n} \log ^{l(n)} Q \cdot \sum_{i} \frac{1}{\psi\left(\xi_{i}\right)^{n}}\right) .
$$

Выбирая $\psi(x)=\phi_{n}(x)^{1 /(n+1)}$, равенство (11) можно переписать в виде

$$
\begin{gathered}
\Phi^{*}(Q, I)=\frac{Q^{n+1}}{2 \zeta(n+1)}|I|+\frac{Q^{n+1}}{2 \zeta(n+1)} \sum_{i} \frac{\phi_{n}\left(\eta_{i}\right)-\phi_{n}\left(\xi_{i}\right)}{\phi_{n}\left(\xi_{i}\right)}\left|I_{i}\right| \\
+O\left(Q^{n} \log ^{l(n)} Q \cdot \sum_{i} \phi_{n}\left(\xi_{i}\right)^{-n /(n+1)}\right) .
\end{gathered}
$$

Обозначая $M_{i}=\sup _{x \in I_{i}} \phi_{n}(x)$ и $m_{i}=\inf _{x \in I_{i}} \phi_{n}(x)$, по лемме 1 получаем

$$
\left|\sum_{i} \frac{\phi_{n}\left(\eta_{i}\right)-\phi_{n}\left(\xi_{i}\right)}{\phi_{n}\left(\xi_{i}\right)}\right| I_{i}|| \leqslant|I| \max _{i} \frac{M_{i}-m_{i}}{m_{i}} \leqslant C|I| \max _{i}\left|I_{i}\right|,
$$

где $C$ - постоянная, зависящая только от $n$. 
Из леммы 1 для суммы в символе $O$ получаем

$$
\sum_{i} \phi_{n}\left(\xi_{i}\right)^{-n /(n+1)} \leqslant R^{2 n /(n+1)} \#\left\{I_{i}\right\}
$$

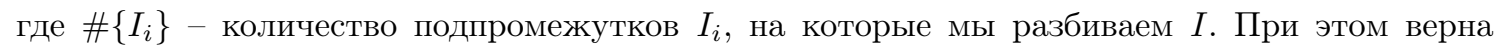
оценка

$$
\#\left\{I_{i}\right\} \leqslant \frac{|I|}{\min _{i}\left|I_{i}\right|} .
$$

Чтобы оба второстепенных члена в (12) оценивались одинаковыми порядками величины, достаточно выполнения условия

$$
\max _{i}\left|I_{i}\right| \cdot \min _{i}\left|I_{i}\right|=\frac{c R^{2 n /(n+1)} \log ^{l(n)} Q}{Q},
$$

где $c$ - постоянная, зависящая только от $n$. Причем наилучшая оценка достигается, когда все подпромежутки $I_{i}$ имеют одинаковые длины:

$$
\max _{i}\left|I_{i}\right|=\min _{i}\left|I_{i}\right|=R^{n /(n+1)} \sqrt{\frac{c \log ^{l(n)} Q}{Q}} .
$$

Отсюда для числа подпромежутков имеем

$$
\#\left\{I_{i}\right\} \leqslant \max \left\{1, \frac{|I|}{R^{n /(n+1)}} \sqrt{\frac{Q}{c \log ^{l(n)} Q}}\right\} .
$$

Таким образом, получаем (10).

Автор выражает благодарность организаторам конференции памяти Анатолия Алексеевича Карацубы (Москва, январь 2016 г.) за прекрасную организацию и теплую атмосферу. Автор благодарит рецензента за ценные замечания.

\section{Список литературы}

[1] A. Walfisz, Weylsche Exponentialsummen in der neueren Zahlentheorie, Mathematische Forschungsberichte, 15, VEB Deutscher Verlag der Wissenschaften, Berlin, 1963.

[2] P. Erdős, M. Kac, E. R. van Kampen, A. Wintner, "Ramanujan sums and almost periodic functions", Studia Math., 9:1 (1940), 43-53.

[3] M. Mikolás, "Farey series and their connection with the prime number problem. I", Acta Univ. Szeged. Sect. Sci. Math., 13 (1949), 93-117.

[4] Л. Кейперс, Г. Нидеррейтер, Равномерное распределение последовательностей, Мир, М., 1985.

[5] J. Franel, "Les suites de Farey et le problème des nombres premiers", Nachr. Ges. Wiss. Göttingen. Math.-Phys. Kl., 1924 (1924), 198-201.

[6] H. Niederreiter, "The distribution of Farey points", Math. Ann., 201:4 (1973), 341-345.

[7] F. Dress, "Discrépance des suites de Farey", J. Théor. Nombres Bordeaux, 11:2 (1999), 345-367.

[8] C. Cobeli, A. Zaharescu, "The Haros-Farey sequence at two hundred years", Acta Univ. Apulensis Math. Inform., 5 (2003), 1-38.

[9] A. Baker, W. M. Schmidt, "Diophantine approximation and Hausdorff dimension", Proc. Lond. Math. Soc. (3), 21:1 (1970), 1-11.

[10] В. И. Берник, "Применение размерности Хаусдорфа в теории диофантовых приближений", Acta Arith., 42:3 (1983), 219-253. 
[11] В. И. Берник, "О точном порядке приближения нуля значениями целочисленных многочленов", Acta Arith., 53:1 (1989), 17-28.

[12] V. Bernik I., M. M. Dodson, Metric Diophantine Approximation on Manifolds, Cambridge Tracts in Math., 137, Cambridge Univ. Press, Cambridge, 1999.

[13] V. Beresnevich, "On approximation of real numbers by real algebraic numbers", Acta Arith., 90:2 (1999), 97-112.

[14] Y. Bugeaud, Approximation by Algebraic Numbers, Cambridge Tracts in Math., 160, Cambridge Univ. Press, Cambridge, 2004.

[15] H. Brown, K. Mahler, "A generalization of Farey sequences: some exploration via the computer", J. Number Theory, 3:3 (1971), 364-370.

[16] Д. У. Каляда, “Аб размеркаванні рэчаісных алгебраічных лікаў дадзенай ступені”, Докл. НАН Беларуси, 56:3 (2012), 28-33.

[17] Д. В. Коледа, “О распределении действительных алгебраических чисел второй степени”, Весці НАН Беларусі. Сер. фіз.-мат. навук, 2013, № 3, 54-63.

[18] D. Kaliada, "On the density function of the distribution of real algebraic numbers", J. Theor. Nombres Bordeaux, 2017 (to appear), arXiv: 1405.1627.

[19] Д.Н. Запорожец, “Случайные полиномы и геометрическая вероятность”, Докл. РАН, 400:3 (2005), 299-303.

[20] Д. В. Коледа, "Об асимптотике распределения алгебраических чисел при возрастании их высот", Чебышевский сб., 16:1 (2015), 191-204.

[21] V. Beresnevich, V. Bernik, F. Götze, "The distribution of close conjugate algebraic numbers", Compos. Math., 146:5 (2010), 1165-1179.

[22] F. Götze, D. Kaliada, D. N. Zaporozhets, "Correlations between real conjugate algebraic numbers", Чебышевский сб., 16:4 (2015), 90-99.

[23] F. Götze, D. Kaliada, D. Zaporozhets, Correlation Functions of Real Zeros of Random Polynomials, 2015, arXiv: 1510.00025 . 